\title{
Dual specific antitumor effects of Semliki Forest virus-based DNA vector carrying suicide Escherichia coli purine nucleoside phosphorylase gene via Salmonella
}

\author{
ZHI-HAO CHEN ${ }^{1}$, GE-LING HUANG ${ }^{2}$, YA-QIN TU ${ }^{1}$, YU JIANG ${ }^{2}$ and WU-XING DAI ${ }^{1}$ \\ ${ }^{1}$ Department of Biochemistry and Molecular Biology, Tongji Medical College, Huazhong University of Science \\ and Technology, Wuhan; ${ }^{2}$ Maternal and Child Health Hospital of Xiamen, Xiamen, P.R. China
}

Received February 10, 2013; Accepted April 2, 2013

DOI: $10.3892 /$ ijo.2013.1900

\begin{abstract}
The Escherichia coli purine nucleoside phosphorylase/2-fluoro-2-deoxyadenosine (ePNP/F-dAdo) suicide system has demonstrated a powerful killing and bystander effects on tumor cells. However, several drawbacks to this approach remain to be resolved, such as the side-effects and the low efficiency of ePNP-targeted expression. A human telomerase reverse transcriptase promoter-driven Semliki Forest virus-based DNA vector (pShT-ePNP) with high expression of the ePNP gene was constructed. Live attenuated Salmonella typhimurium 7207 (SL7207) was used initially as a vehicle to targetly transfer the large alphavirus vector into tumor cells. The in vitro quantitative analysis showed 2 -fold higher green fluorescent protein (GFP) expression for pShT-GFP than for conventional cytomegalovirus (CMV) promoter-mediated eukaryotic expression plasmids such as pIRES-GFP and the targeted expression of the ePNP gene in tumor cells was also detected by RT-PCR. After F-dAdo addition, the enzymatic conversion of F-Ado into 2-fluoroadmine (F-Ade) was tested by HPLC. Cell cytotoxicity assays showed that the significant inhibitory effect of the SL/pShT-ePNP system on tumor cells was dose- and time-dependent. Following oral administration, recombinant bacteria targetly allocated within the solid tumor and the expression of ePNP and GFP genes in vivo were detected by RT-PCR or observed by fluorescence microscopy. SL/pShT-ePNP and F-dAdo were also found to exert powerful therapeutic effects in combination against tumor growth and for prolonging the lifespan of tumor-bearing mice. These find-
\end{abstract}

Correspondence to: Professor Wuxing Dai, Department of Biochemistry and Molecular Biology, Tongji Medical College, Huazhong University of Science and Technology, 13 Hangkong Road, Wuhan 430030, P.R. China

E-mail: daiwuxing2002@163.com

Key words: human telomerase reverse transcriptase promoter, Semliki Forest virus, Salmonella typhimurium, Escherichia coli purine nucleoside phosphorylase, 2-fluoro-2-deoxyadenosine, DNA vector, tumor therapy ings suggest that the SL/pShT-ePNP system may serve as a powerful strategy for tumor therapy.

\section{Introduction}

The Escherichia coli purine nucleoside phosphorylase/2fluoro-2-deoxyadenosine suicide system has been found to have powerful killing and bystander effects on tumor cells (1). However, several drawbacks to cancer suicide/gene therapy remain to be resolved, including the side-effects of this therapy (2). The human telomerase reverse transcriptase (hTERT) promoter has been widely used to drive the specific expression of therapeutic genes for the treatment of tumor cells $(3,4)$. However, the transcriptional activity of the hTERT promoter is weaker than that of the conventional CMV promoter, which results in an insufficient therapeutic effect (5).

The pSFV1 eukaryotic expression vector (Invitrogen, USA), which is based on the Semliki Forest virus (SFV) replicon, is a self-replicating RNA vector with a high level of expression efficiency (6). After transfection of the SFV-based DNA vector, an initial plus-strand full-length RNA driven by the CMV promoter is transcribed in the nucleus, translocated into the cytoplasm and then translated into the replicase complex of SFV. The replicase complex directly initiates the replication cascade and consequently, high-level transcription of exogenous genes occurs in the cytoplasm. Death is induced in host cells transfected by this SFV-based DNA vector and a large amount of the protein expressed from the vector is released, which eliminates the potential genotoxic risks of exogenous DNA (7-9). Studies have shown that protein expression based on the alphavirus replicase complex is several times greater than the protein expression driven by the conventional CMV promoter (8). However, the transfection efficiency of the plasmid is significantly reduced because its size is $\sim 12 \mathrm{~kb}$; hence, its application in disease research has been greatly limited (10).

Several types of anaerobes have great potential as vectors for carrying plasmids into cells (11-13). In previous studies, attenuated Salmonella typhimurium SL7207 was used as an effective vehicle for transporting plasmids into cells. Because this organism is an aroA-defective anaerobe that exhibits limited proliferation within cells, the plasmids are released 
upon the death of the bacterium, which results in high expression of the exogenous genes $(14,15)$.

Therefore, in this study, we designed and constructed a new SFV-based DNA vector carrying a replicase gene under the control of the hTERT promoter, which ensure targeted and powerful gene expression in tumor cells. To our knowledge, this study is the first to use transfer of this big plasmid into tumor cells with SL7207 as a vehicle to achieve high levels of expression of the ePNP gene in the cytoplasm. We expect that administration of this live recombinant bacterial vaccine together with the prodrug F-dAdo could provide a new strategy for clinical therapy of solid tumors.

\section{Materials and methods}

Animals, bacterial strains, plasmids and cells. Female C57BL/6J mice (age 6-8 weeks) and a feeding site were provided by the Laboratory Animal Center of Xiamen University. The attenuated S. typhimurium strains LB5000 and SL7207 were obtained from ATCC (Rockville, MD, USA). The plasmid pSFV1 was generously provided by Professor Zhuozhuang Lu (Chinese Center for Disease Control and Prevention, China). The plasmids pGL3-hTERT-luc, pCI-neo, pIRES and pIRES-GFP were provided by Dr Hanbing $\mathrm{He}$ (School of Life Sciences, Sichuan University, China). Murine B16 and LLC cells and human WI-38 cells were purchased from the Shanghai Cell Bank of the Chinese Academy of Science and grown in Dulbecco's modified Eagle medium (DMEM) with 10\% FBS and antibiotic-antimycotic mix (Gibco BRL, USA).

Vector construction and transformation of S. typhimurium with plasmids. The plasmid pSFV1 was used as the basic plasmid. The hTERT promoter sequence was amplified from plasmid pGL3-hTERT-luc and used to replace the SP6 promoter in plasmid pSFV1 by overlapping polymerase chain reaction $(\mathrm{PCR})$. A strong transcription-termination signal, the SV40 poly(A) sequence amplified from plasmid pCI-neo was inserted downstream of the multiple cloning site by overlap PCR and the restriction endonuclease sites BamHI and ClaI were introduced into the multiple cloning site simultaneously. The plasmid pShT was then constructed successfully. The ePNP and GFP genes were amplified from the Escherichia coli genome and the plasmid pIRES-GFP, respectively and cloned into the multiple cloning site of the plasmid pShT to construct the plasmids pShT-ePNP and pShT-GFP (Fig. 1A). Similarly, the ePNP gene was cloned into plasmid pIRES to construct the plasmid pIRES-ePNP. All recombinant plasmids were analyzed by restriction enzyme digestion and sent for sequencing to Shanghai Biological Engineering Co. (SBEC, China). These plasmids were then transformed into attenuated $S$. typhimurium LB5000 by electroporation $(12.5 \mathrm{kV}$, 1 impulse, $4.8 \mathrm{~ms}$ ) by using a Gene Pulser II apparatus (Bio-Rad, USA). The plasmids were obtained from the positive clones and then introduced into S. typhimurium SL7207 under the same conditions used for LB5000. The recombinant bacteria were identified as SL/pShT, SL/pShT-ePNP, SL/ pIRES-ePNP, SL/pShT-GFP and SL/pIRES-GFP and were amplified in $\mathrm{LB}$ medium and stored at $-80^{\circ} \mathrm{C}$ for subsequent experiments.
Bacterial infection and gene expression. Recombinant bacteria $(20 \mu \mathrm{l}) \mathrm{SL} / \mathrm{pShT}$, SL/pShT-GFP and SL/pIRES-GFP were seeded into $200 \mathrm{ml}$ of LB medium containing ampicillin $(100 \mu \mathrm{g} / \mathrm{ml})$ and were grown at $37^{\circ} \mathrm{C}$ for $16 \mathrm{~h}$. The bacterial count was adjusted to $2 \times 10^{8} \mathrm{cfu} / \mathrm{ml}$ by using an automatic urinary sediment analyzer (Sysmex, Japan). Murine B16 cells and LLC cells and human WI-38 cells $\left(2 \times 10^{5}\right.$ cells/well) were seeded into 6-well plates until the cells reached $70-80 \%$ confluence. Next, $100 \mu \mathrm{l}$ of recombinant bacteria was added when the multiplicity of infection (MOI) was 100. After incubation for $2 \mathrm{~h}$, the cells were washed twice and new medium containing tetracycline $(10 \mathrm{mg} / \mathrm{l})$ was added for further culturing. Simultaneously, $1 \mu \mathrm{mol}$ of plasmid pIRES-GFP or pShTGFP was transfected into cells using Lipofectamine 2000 (Lipo2000, Invitrogen). Forty-eight hours later, the expression efficiency of GFP was observed by fluorescence microscopy and the expression level was quantitatively analyzed according to the handling procedures for the GFP quantification kit (Biovision, USA). Forty-eight hours after infection with SL/ pShT, SL/pShT-ePNP and SL/pIRES-ePNP, the infected cells were harvested and ePNP gene expression was monitored by reverse transcriptase-polymerase chain reaction (RT-PCR).

Efficiency of F-dAdo conversion to F-Ade. To determine the efficiency of F-dAdo (Sigma, USA) conversion to 2-fluoroadenine (F-Ade), the cells $\left(2 \times 10^{5}\right.$ cells/well) were seeded onto 6 -well plates. Twenty-four hours after infection, the medium was changed and F-dAdo was added until a final concentration of $80 \mu \mathrm{g} / \mathrm{ml}$ was achieved. After another $48 \mathrm{~h}$, the cell supernatant was harvested for boiling, followed by centrifugation at $12,000 \mathrm{~g}$ for $5 \mathrm{~min}$. The supernatant was again harvested and then analyzed by high-performance liquid chromatography (HPLC) (16).

Cytotoxicity assays. B16, LLC and WI-38 cells were infected with recombinant bacteria at various MOIs. Twenty-four hours after infection, F-dAdo was added until a final concentration of $80 \mu \mathrm{g} / \mathrm{ml}$ was achieved. Seventy-two hours after F-Ado addition, the cell counting kit-8 (CCK-8, Dojindo, Japan) was used. The optical density (OD) difference was measured $1 \mathrm{~h}$ later at $450 \mathrm{~nm}$. The relative survival rate of cells was calculated using the following formula: $\left(\mathrm{A}_{\text {sample }}-\mathrm{A}_{\text {blank }}\right) /\left(\mathrm{A}_{\text {control }}-\mathrm{A}_{\text {blank }}\right) \times 100 \%$ (17). The relative survival rates of infected cells $(\mathrm{MOI}=100)$ at different F-Ado concentrations $(0-160 \mu \mathrm{g} / \mathrm{ml})$ were observed by using the CCK-8 as described above. Similarly, following incubation with $80 \mu \mathrm{g} / \mathrm{ml} \mathrm{F-dAdo,} \mathrm{the} \mathrm{relative} \mathrm{survival} \mathrm{rates} \mathrm{of}$ infected cells $(\mathrm{MOI}=100)$ at different time-points were also calculated as described above.

In vivo gene expression and analysis of antitumor effects. C57BL/6J mice were used according to the guidelines for administration to lab animals, issued by the Ministry of Science and Technology (Beijing, China) and an animal care and use protocol approved by Xiamen University. Tumors were established by subcutaneous inoculation with B16 melanoma cells (100 $\mu \mathrm{l}$ containing $1 \times 10^{6}$ cells). When the tumor volume reached $100 \mathrm{~mm}^{3}$, the following experiments were conducted. Four B16 melanoma-bearing mice were sacrificed 4 days after oral administration of $5 \times 10^{7} \mathrm{cfu}$ of SL/pIRESGFP, SL/pShT-GFP, or SL/pShT and phosphate-buffered 
A

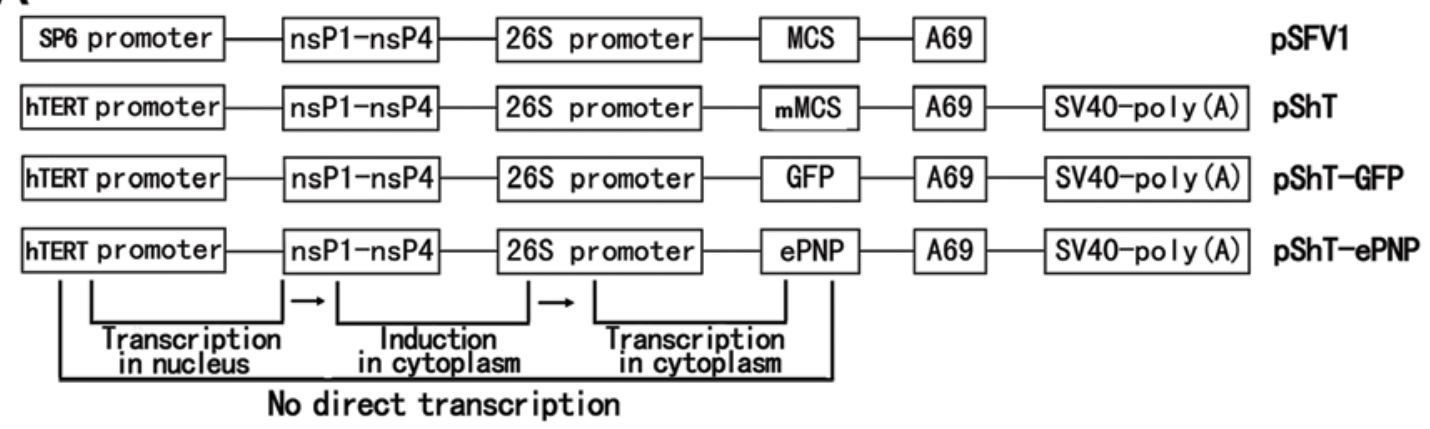

B
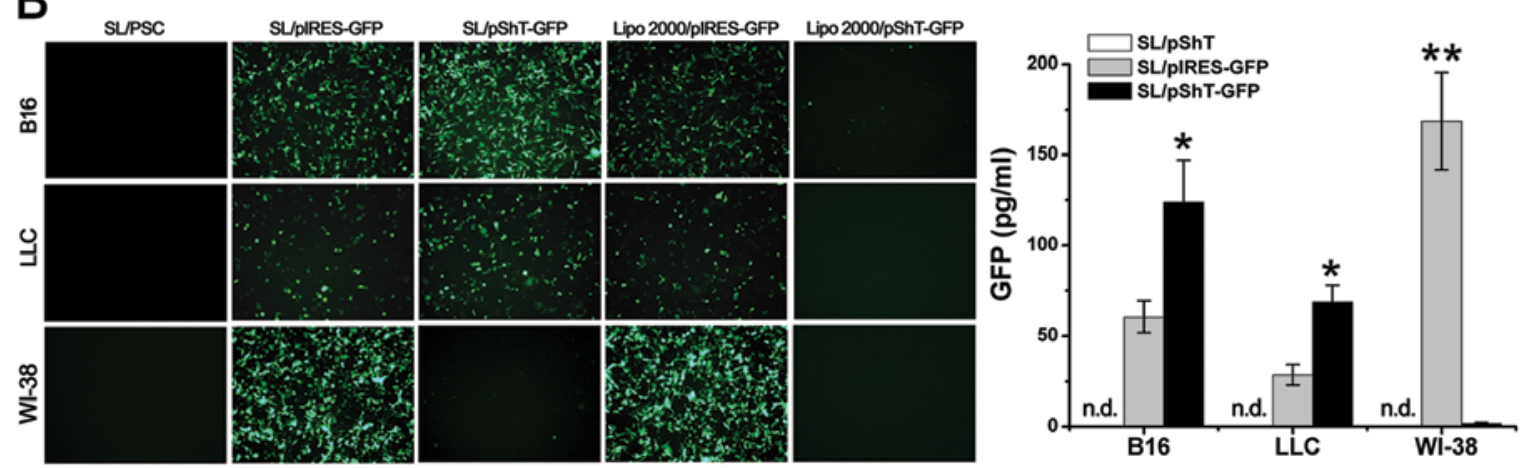

Figure 1. Vector construction design and functional examination of the DNA vaccine plasmid. (A) DNA fragments encoding GFP or ePNP were obtained by PCR and inserted into plasmid pShT, which was modeled on plasmid pSFV1. (B) B16, LLC and WI-38 cells were infected by SL/pShT, SL/pIRES-GFP or SL/ pShT-GFP and $1 \mu \mathrm{mol}$ of plasmid pIRES-GFP or pShT-GFP was transfected into cells by using Lipofectamine 2000. Forty-eight hours later, GFP expression was determined by fluorescence microscopy. The level of GFP expression was examined using a GFP quantification kit. The data are the mean \pm SD values of 3 separate experiments. ${ }^{~} \mathrm{P}<0.05$ versus SL/pIRES-GFP. ${ }^{* * *} \mathrm{P}<0.01$ versus $\mathrm{SL} / \mathrm{pShT}$-GFP. ND, not detectable.

saline (PBS) (as a control). Tumor tissues were excised and cut into frozen sections for the detection of GFP expression. B16 melanoma-bearing mice were divided into 5 groups of 13 mice each. The mice in each group were orally administered $1 \mathrm{ml}$ PBS containing $5 \times 10^{7} \mathrm{cfu}$ of SL, SL/pShT, SL/pIRES-ePNP, or SL/pShT-ePNP or were administered $1 \mathrm{ml}$ PBS as the control. F-dAdo $(10 \mathrm{mg} / \mathrm{kg})$ dissolved in $0.5 \mathrm{ml}$ PBS was injected intraperitoneally 3 times daily for 3 consecutive days, beginning at $48 \mathrm{~h}$ after the administration of recombinant bacteria. This schedule was counted as a single course and 3 consecutive courses were administered (18). Tumor diameters were measured using calipers every 2 days and tumor volumes were calculated using the following formula: volume $=$ length $\mathrm{x}$ width ${ }^{2} \mathrm{x} 0.52$. On the fourth day of the first course, 3 mice in each group were sacrificed and tumor and other organs were excised. The expression of ePNP gene in tumors for each group was examined by RT-PCR. Simultaneously, cell suspensions prepared from the tumor, heart, liver, spleen and lung in SL/pShT, SL/pShT-ePNP and SL/pIRES-ePNP groups were spread on LB agar plates containing ampicillin $(100 \mu \mathrm{g} /$ $\mathrm{ml}$ ) to analyze the bacterial distribution in vivo. At the end of the third course, 5 mice in each group were sacrificed and the tumor specimens were subjected to histopathological analysis and TUNEL staining (Roche, Switzerland).

To evaluate the specificity of this therapeutic vaccine, on the fourth day of the first course, the expression of the ePNP gene in tumor tissue and various organs of B16 tumor-bearing mice in SL/pShT group $(n=3)$ was detected by RT-PCR assays. To evaluate the safty of this therapeutic vaccine, peripheral blood was drawn from the orbital region of 5 mice in each group at the end of the third course for biochemical and hematological assays performed using a DXC 800 biochemical auto-analyzer (Beckman, USA) and XS-1000i hematology analyzer (Sysmex, Japan). The heart, liver, spleen and lungs were also subjected to histopathological analysis for safty evaluation. When the tumor size reached $4,500 \mathrm{~mm}^{3}$, the other 5 B16 melanomabearing mice from each group were sacrificed and the time until sacrifice was defined as the survival time.

Statistical analysis. One-way analysis of variance (ANOVA) was used to evaluate the experimental data and the groups were compared using Dunnet's t-test. The log-rank test was used to analysis the survival times of the mice. Differences were considered significant at $\mathrm{P}<0.05$.

\section{Results}

In vitro expression of exogenous genes of the pShT plasmid carried by SL7207. A series of plasmids was constructed using commercialized pSFV-1 as a template. Sequencing confirmed that all plasmids were successfully constructed. B16, LLC and WI-38 cells were infected with SL/pShT, SL/pShT-GFP, or SL/ 


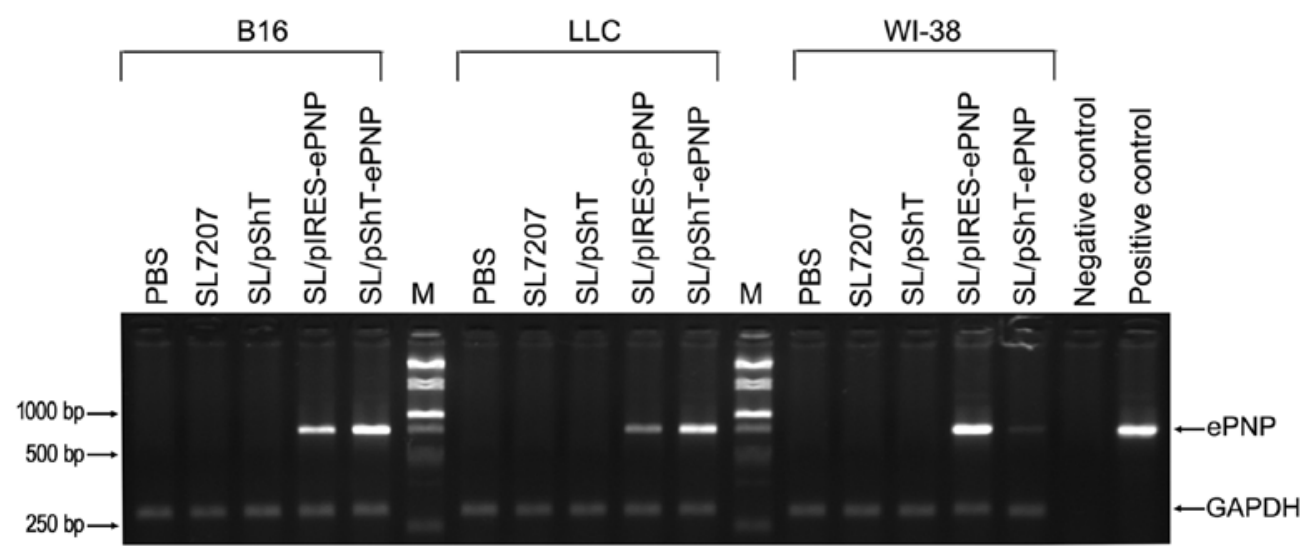

Figure 2. RT-PCR analysis of ePNP gene expression. Forty-eight hours after infection with recombinant bacterial, the infected cells were harvested and expression of the ePNP gene was monitored by RT-PCR.

pIRES-GFP and transfected with the plasmids pIRES-GFP and pShT-GFP by using Lipofectamine 2000. Forty-eight hours later, fluorescence microscopy analysis helped detect high levels of GFP expression from B16 and LLC cells in the SL/ pIRES-GFP and SL/pShT-GFP groups, whereas WI-38 cells showed detectable GFP expression only in the SL/pIRES-GFP group and almost no GFP expression in the SL/pShT-GFP group, which indicates that plasmid pShT was tumor-targeted (Fig. 1B). We also observed a high level of GFP expression in the Lipo2000/pIRES-GFP group for B16 and LLC cells, whereas almost no GFP expression was detected for the Lip2000/pShT-GFP group (Fig. 1B). Quantitative analysis showed the highest GFP expression in the SL/pShT-GFP group, which was $\sim 2.0$ and 2.4 times higher than that of the SL/pIRES-GFP group for B16 cells and LLC cells, respectively (Fig. 1B, P<0.05). Total RNA was extracted from the infected cells and ePNP mRNA was detected by RT-PCR. A band was detected at $750 \mathrm{bp}$ in the SL/pIRES-ePNP and SL/ pShT-ePNP groups for B16 and LLC cells, whereas almost no expression was detected for WI-38 cells (Fig. 2). These results demonstrated that a series of constructed plasmids based on pSFV-1 could be effectively transported into tumor cells by SL7207 and expressed there in a targeted manner. Moreover, the expression efficiency was considerably better than that of traditional eukaryotic expression vectors such as pIRES.

Effects of F-dAdo on B16 cells infected by SL7207 carrying various plasmids. To observe the effect of the prodrug F-dAdo on tumor cells, we used HPLC to monitor the ratio of F-dAdo conversion. Forty-eight hours after F-dAdo addition, the ratio of F-dAdo conversion for B16 and LLC cells in the SL/pShTePNP group reached 46.57 and $28.40 \%$, respectively, whereas that of the SL/pIRES-PNP group was only 26.37 and $12.26 \%$, respectively. Significant differences were observed between the SL/pShT-ePNP group and the SL/pIRES-PNP group for B16 and LLC cells (Fig. 3A, P<0.05). Moreover, WI-38 cells in the SL/pShT-ePNP group showed no sensitivity to F-Ado, which indicates that the plasmid pShT-ePNP was only functional in tumor cells.

We used different concentrations of recombinant bacteria to infect cells. In the experiment on B16 cells, when the MOI was 0.1 and 10 , the cell viability in the SL/pShT-ePNP group was 47.65 and $13.45 \%$, respectively $(\mathrm{P}<0.05)$ and that in the SL/pIRES-PNP group was 62.65 and $17.68 \%$, respectively $(\mathrm{P}<0.05)$. Similar results were obtained for LLC cells. In contrast, in the control WI-38 cells, the cell viability in the $\mathrm{SL} / \mathrm{pShT}$-ePNP group did not differ with increase in the MOI (Fig. 3B). We used the half-inhibitory concentration $\left(\mathrm{IC}_{50}\right)$ to evaluate the cytotoxicity of the suicide gene system. After addition of different concentrations of F-Ado, the B16 cells in the SL/pShT-ePNP and SL/pIRES-ePNP groups showed significantly decreased viability whereas those in the PBS, SL and SL/pShT groups did not appear to be affected. The lowest $\mathrm{IC}_{50}$ was seen in the SL/pShT-ePNP group $(6.86 \mu \mathrm{g} / \mathrm{ml})$ and the $\mathrm{IC}_{50}$ of the SL/pIRES-ePNP group was $12.34 \mu \mathrm{g} / \mathrm{ml}(\mathrm{P}<0.05)$. Similar results were also obtained for LLC cells. In contrast, in the control WI-38 cells, there was no significant cytotoxicity in any group except for the SL/pIRES-PNP group (Fig. 3C). When the MOI of the recombinant bacteria was 100 and the concentration of F-Ado was $80 \mu \mathrm{g} / \mathrm{ml}$, it was observed that as the treatment time increased, the cell viability for B16 and LLC cells in the SL/pShT-ePNP group decreased, whereas no such result was observed for the control WI-38 cells (Fig. 3D). These results indicate that the plasmid pShT-ePNP can be effectively delivered into cells by SL7207, expressed specifically in tumor cells and exert cytotoxicity when F-Ado is added. Furthermore, the cytotoxicity observed increased over time and was concentration-dependent.

In vivo expression of ePNP in tumors in $B 16$ melanoma-bearing mice. Four tumor-bearing mice were orally administered PBS, $\mathrm{SL} / \mathrm{pShT}$, SL/pShT-GFP, or SL/pIRES-GFP and sacrificed 4 days later. The tumors were immediately excised, sectioned and frozen. High levels of GFP expression were observed in the SL/pShT-GFP or SL/pIRES-GFP group on fluorescence microscopy analysis. The PBS and SL/pShT groups were used as the controls (Fig. 4A). Tumor tissues of the treated mice were dispersed into cell suspensions and total RNA was extracted for RT-PCR analysis. Bands were observed at 750 bp in the SL/pShT-ePNP and SL/pIRES-ePNP groups, which indicates that ePNP was successfully expressed in tumor tissue (Fig. 4B). These results demonstrate that the plasmid constructed, that is, pShT-ePNP was transferred 

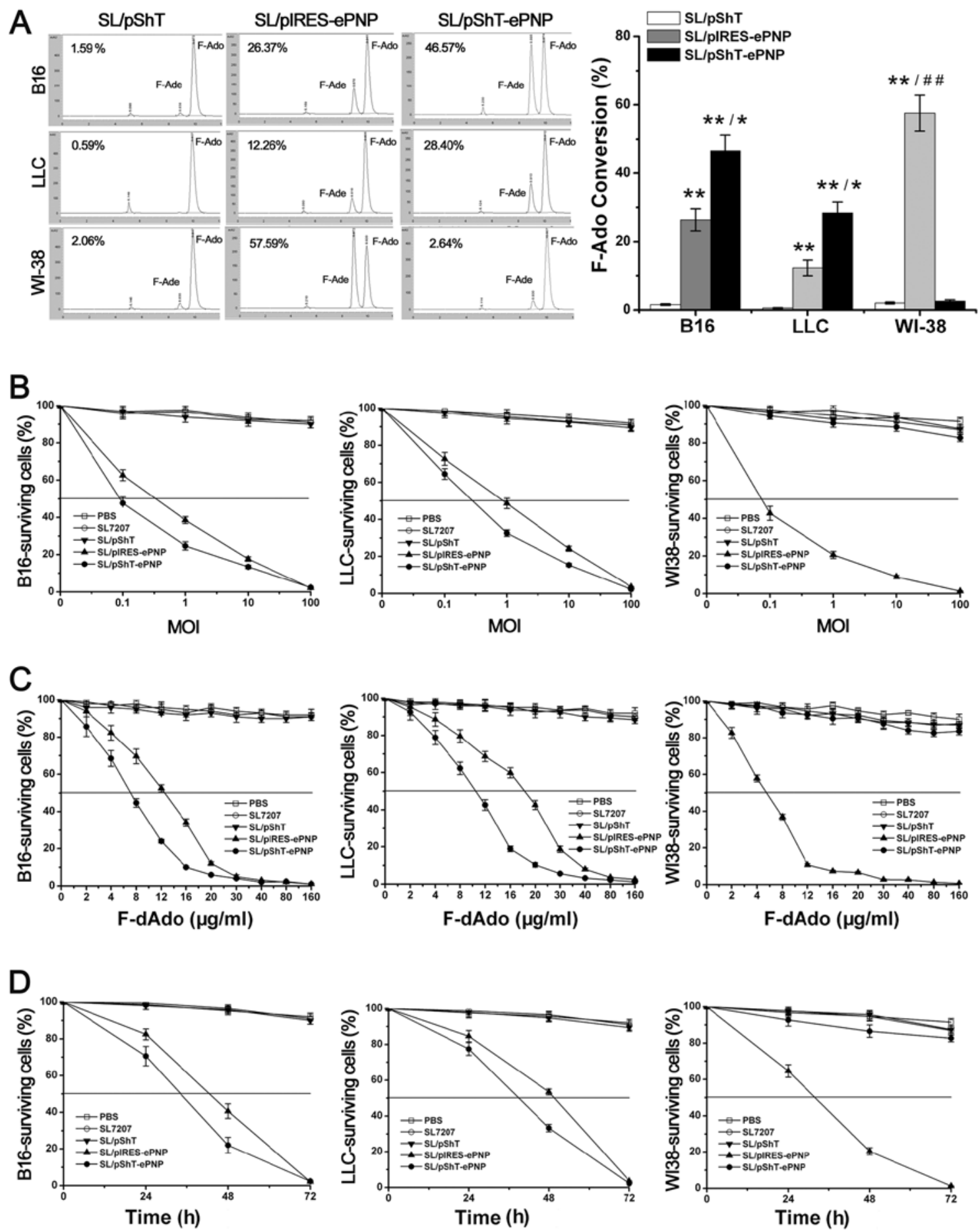

Figure 3. Effects of infection by various recombinant bacteria in B16, LLC and WI-38 cells after addition of F-dAdo. (A) The infected cells were cultured with $80 \mu \mathrm{g} / \mathrm{ml} \mathrm{F-dAdo} \mathrm{in} \mathrm{6-well} \mathrm{plates.} \mathrm{Forty-eight} \mathrm{hours} \mathrm{later,} \mathrm{the} \mathrm{efficiency} \mathrm{of} \mathrm{F-dAdo} \mathrm{conversion} \mathrm{to} \mathrm{F-Ade} \mathrm{was} \mathrm{determined} \mathrm{by} \mathrm{HPLC} \mathrm{at} 260 \mathrm{~nm}$. The data provided are the mean \pm SD values of 3 separate experiments. ${ }^{* *} \mathrm{P}<0.01$ versus $\mathrm{SL} / \mathrm{pShT}$ group. ${ }^{*} \mathrm{P}<0.05$ versus $\mathrm{SL} / \mathrm{pIRES}$-ePNP group. ${ }^{\# \# /} \mathrm{P}<0.01$ versus SL/pShT-ePNP group. (B) Cells were infected with recombinant bacterial at various MOIs. Twenty-four hours after infection, F-dAdo was added at a final concentration of $80 \mu \mathrm{g} / \mathrm{ml}$. Seventy-two hours after F-Ado addition, CCK-8 was used and the absorbance of each well was measured at $570 \mathrm{~nm}$. The data provided are the mean \pm SD values of 3 separate experiments and are expressed as percentages relative to values for the untreated cells. (C) The infected cells $(\mathrm{MOI}=100)$ were cultured with various concentrations of F-dAdo $(0-160 \mu \mathrm{g} / \mathrm{ml})$ in 96-well plates. Three days later, the survival of infected cells was measured using CCK-8 as described above. (D) Following incubation with $80 \mu \mathrm{g} / \mathrm{ml} \mathrm{F-dAdo,} \mathrm{the} \mathrm{survival} \mathrm{of} \mathrm{infected} \mathrm{cells} \mathrm{(MOI}=100)$ at different time-points was quantified using CCK-8 as described above.

into tumor cells by oral administration of SL7207 and was expressed successfully in vivo.

Therapeutic efficacy of SL/pShT-ePNP for B16 melanomabearing mice. After oral administration of recombinant bacteria and intraperitoneal injection of F-dAdo, tumor volume was measured every 2 days and the data were used to draw a tumor growth curve (Fig. 5B). No significant differences were observed among groups at the beginning of treatment 

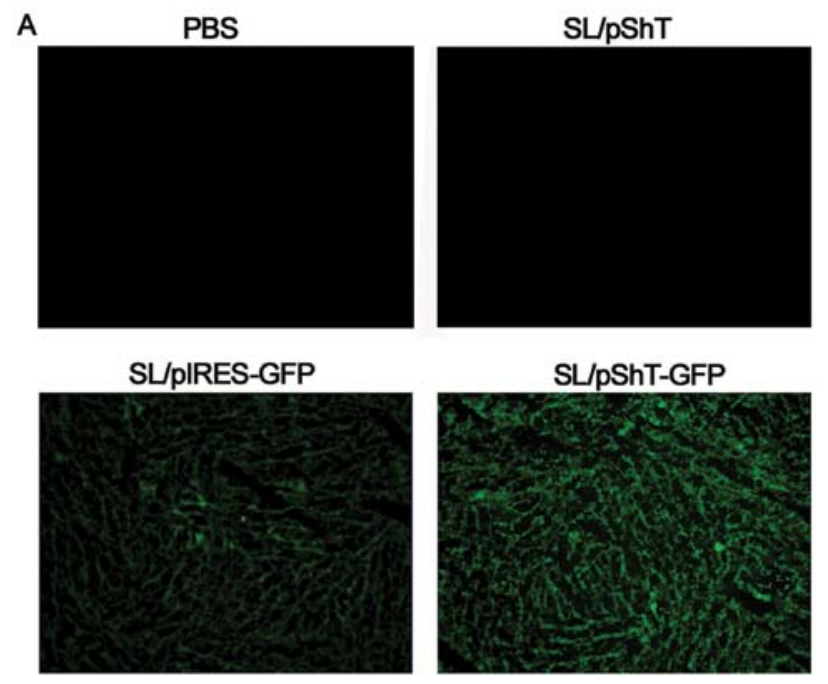

B

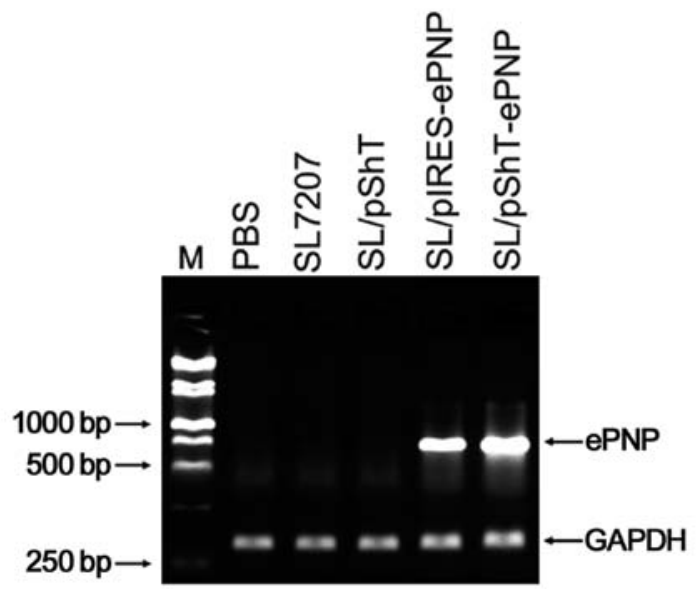

Figure 4. Delivery of recombinant bacteria carrying various plasmids and expression of GFP and ePNP genes in vivo. (A) Four B16 melanoma-bearing mice were orally administered with SL/pIRES-GFP and SL/pShT-GFP and the tumor tissues were prepared as frozen sections 4 days later. The GFP signal in the tumor tissues was captured by fluorescence microscopy. Animals treated with PBS and SL/pShT were used as the control group. (B) Tumor tissues of the mice in each group $(n=3)$ were dispersed into cell suspensions. ePNP mRNA expression in mouse tumor tissues was examined by RT-PCR.
( $\mathrm{P}>0.05)$. Three days after the entire treatment was completed, a pairwise comparison was performed among these groups. The average volumes of tumors from the SL/pIRES-ePNP and SL/pShT-ePNP groups were significantly lower than the volumes of tumors from the other groups. Tumors from the SL/pShT-ePNP group exhibited the lowest volume. There were significant differences between the SL/pShT-ePNP group and the SL/pIRES-ePNP group $(\mathrm{P}<0.05)$. A survival analysis curve for B16 tumor-bearing mice is shown in Fig. 5C. The survival time of each group was analyzed by a log-rank test and the results indicated significant differences among the groups. The survival time of the SL/pShT-ePNP group was significantly greater than that of the other groups. The pathological results showed large-scale coagulation necrosis and apoptotic cells in the tumor tissues of the SL/pShT-ePNP group and this phenomenon was also seen in other groups to different degrees except in the PBS control group, in which no obvious changes were seen (Fig. 6). Thus, the SL/pShT-ePNP vaccine was effective in treating B16 melanoma-bearing mice and prolonging their survival.

Target specificity and safety analysis of SL/pShT-ePNP therapeutic vaccine. Tumor tissue and various important organs of B16 melanoma-bearing mice that were orally administered recombinant bacteria were dispersed into cell suspensions and cultured on LB plates. The bacterial concentrations in the heart, liver, spleen and lung were very low. The concentration of bacteria in the SL/pShT-ePNP group was very high in tumors $\left(\leq 7.8 \times 10^{7} \mathrm{cfu} / \mathrm{ml}\right)$ and the ratio of the concentration in the tumors to that in the liver was $\sim 27,000: 1$ (Fig. 7A, $\mathrm{P}<0.01$ ). The RT-PCR results for tumors and organs in the SL/pShTePNP group showed that expression of the ePNP gene was only present in tumors; expression of the ePNP gene could not be detected in other tissues (Fig. 7B). Pathological analyses of various important organs were performed; no significant pathological changes were detected (Fig. 7C). Neither SL/ pShT-ePNP-treated mice nor control mice had any abnormal results for the blood tests or liver function tests (Table I),

Table I. Biochemical and hematological analyses.

\begin{tabular}{lccccc}
\hline Items & PBS & SL7207 & SL/pShT & SL/pIRES-ePNP & SL/pShT-ePNP \\
\hline TP $(\mathrm{g} / \mathrm{l})$ & $57.55 \pm 3.89$ & $55.68 \pm 3.55$ & $57.20 \pm 4.02$ & $56.72 \pm 3.80$ & $57.23 \pm 4.12$ \\
ALB $(\mathrm{g} / \mathrm{l})$ & $30.25 \pm 5.23$ & $31.27 \pm 4.86$ & $31.43 \pm 5.08$ & $32.72 \pm 4.79$ & $32.58 \pm 4.25$ \\
GOT $(\mathrm{U} / \mathrm{l})$ & $41.3 \pm 21.7$ & $42.5 \pm 20.5$ & $42.8 \pm 21.6$ & $43.8 \pm 22.7$ & $40.0 \pm 20.7$ \\
GPT $(\mathrm{U} / \mathrm{l})$ & $123.8 \pm 21.8$ & $120.6 \pm 22.9$ & $122.4 \pm 23.7$ & $125.7 \pm 24.2$ & $124.7 \pm 23.1$ \\
GLU $(\mathrm{mmol} / \mathrm{l})$ & $3.65 \pm 0.42$ & $3.72 \pm 0.45$ & $3.53 \pm 0.48$ & $3.74 \pm 0.56$ & $3.76 \pm 0.49$ \\
BUN $(\mathrm{mmol} / \mathrm{l})$ & $8.59 \pm 1.38$ & $8.67 \pm 1.20$ & $8.53 \pm 1.23$ & $8.78 \pm 1.30$ & $8.74 \pm 1.32$ \\
CREA $(\mu \mathrm{mmol} / \mathrm{l})$ & $38.42 \pm 4.37$ & $38.64 \pm 4.68$ & $39.52 \pm 4.88$ & $38.77 \pm 5.99$ & $37.56 \pm 4.60$ \\
WBC $\left(10^{9} / \mathrm{l}\right)$ & $8.42 \pm 2.96$ & $11.37 \pm 3.50$ & $12.03 \pm 4.05$ & $13.20 \pm 3.46$ & $12.47 \pm 4.63$ \\
RBC $\left(10^{9} / \mathrm{l}\right)$ & $7.30 \pm 0.78$ & $7.18 \pm 0.63$ & $6.89 \pm 0.56$ & $7.02 \pm 0.74$ & $7.20 \pm 0.67$ \\
HGB $(\mathrm{g} / \mathrm{l})$ & $122.35 \pm 18.42$ & $127.59 \pm 17.93$ & $130.57 \pm 22.04$ & $129.58 \pm 19.50$ & $132.47 \pm 17.88$ \\
PLT $\left(10^{9} / \mathrm{l}\right)$ & $538.95 \pm 150.37$ & $582.32 \pm 163.24$ & $576.37 \pm 153.47$ & $566.49 \pm 132.57$ & $542.70 \pm 148.32$ \\
\hline
\end{tabular}

TP, total protein; ALB, albumin; GOT, glutamic oxaloacetic transaminase; GPT, glutamic pyruvic transaminase; GLU, glucose; BUN, blood urea nitrogen; CREA, creatinine; WBC, white blood cell; RBC, red blood cell; HGB, hemoglobin; PLT, plastocyte. 

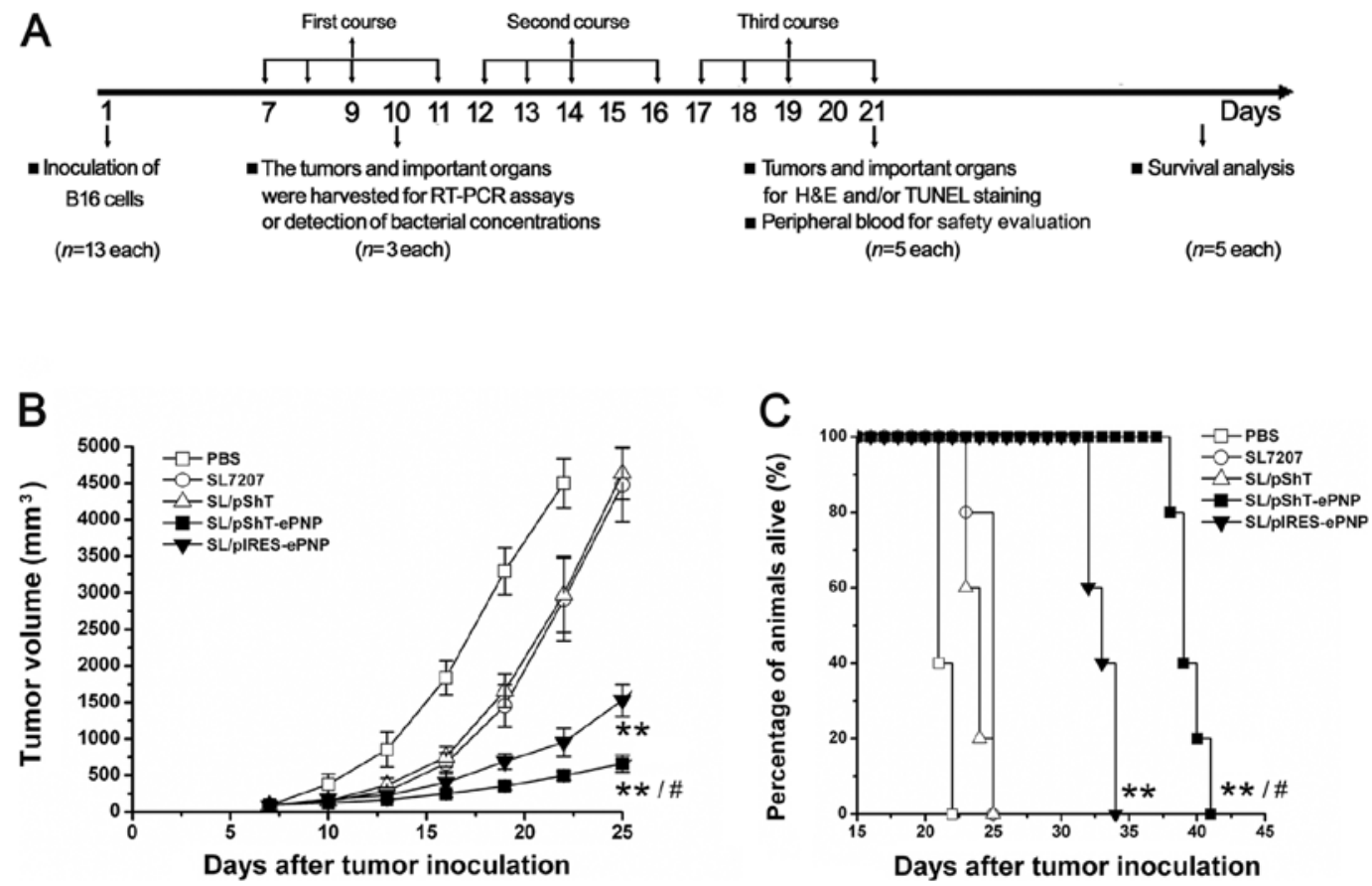

Figure 5. Tumor regression in tumor-bearing mice following oral administration of recombinant bacteria. (A) When the mean tumor volume reached $100 \mathrm{~mm}^{3}$, mice in each group ( $\mathrm{n}=10$ ) were orally administered $1 \mathrm{ml}$ of PBS containing various recombinant bacteria or $1 \mathrm{ml}$ of PBS as a control. F-dAdo (10 mg/kg) dissolved in $0.5 \mathrm{ml}$ PBS was injected intraperitoneally 3 times daily for 3 consecutive days, beginning at 2 days after administration of recombinant bacteria This schedule was counted as a single course and 3 consecutive courses were administered. (B) The mean tumor volume reached $100 \mathrm{~mm}^{3}$ prior to the initial oral administration for every group $(\mathrm{n}=5)$. Tumor volumes were measured every 2 days. Average tumor volumes are shown for the indicated time-points. ${ }^{* *} \mathrm{P}<0.01$ versus $\mathrm{SL} / \mathrm{pShT}$ group, ${ }^{*} \mathrm{P}<0.05$ versus SL/pIRES-ePNP group. (C) Survival curves of B16 melanoma-bearing mice for the different groups ( $\mathrm{n}=5$ ). Statistical differences were evaluated using the log-rank test. ${ }^{* *} \mathrm{P}<0.01$ versus the $\mathrm{PBS}$ or $\mathrm{SL} / \mathrm{pShT}$ group; ${ }^{\mathrm{P}} \mathrm{P}<0.05$ versus SL/pIRES-ePNP group.

which indicates that the SL/pShT-ePNP therapeutic system is safe and effective for tumor treatment.

\section{Discussion}

To our knowledge, our study is the first to combine a targeted virus replicon vector with attenuated S. typhimurium for tumor treatment. We cloned the suicide gene ePNP into the targeted alphavirus replicon-based vector $\mathrm{pShT}$ to express the ePNP gene with high efficiency and enhanced target specificity in tumor cells. When the prodrug F-Ado was added, massive cytotoxicity was induced. Both in vitro and in vivo experiments confirmed the efficiency and specificity of this system.

Previous studies on suicide gene therapy for tumors have often used the hTERT promoter to target the therapeutic effect to tumor cells $(19,20)$. However, all the tumor-specific promoters had poor expression efficiency that limited the expression of the suicide gene in tumor cells and affected the efficacy of these therapies (5). Therefore, alphavirus replicon was induced to improve the expression efficacy of the hTERT promoter. We constructed the alphavirus repliconbased vectors pShT-ePNP and pShT-GFP. However, when we transfected the plasmid pShT-GFP using Lipofectamine 2000, almost no fluorescence was seen after $48 \mathrm{~h}$, which indicates very low transfection efficiency. We speculated that the size of pShT-GFP reaches $12 \mathrm{~kb}$; therefore, conventional transfection agents may not be able to transfect it into cells with sufficient efficiency (21). Thus, we had to find some other way to effectively solve this problem.
The anaerobic attenuated S. typhimurium SL7207, which was engineered to knock out the aroA gene, grows in clusters in tumor tissues and invades tumor cells $(22,23)$. However, these bacteria cannot grow in tumor cells for a long time and when they eventually die within the cell, they release their contents into the cell, including plasmids that can be transcribed and expressed in the cell $(24,25)$. Therefore, we used electrotransformation to place pShT-GFP and pShT-ePNP plasmids into attenuated $S$. typhimurium that would then carry the plasmid into tumor cells (26). Theoretically, after the vector is transferred into host cells, the hTERT promoter controls the transcription of the alphavirus replicon, which is further translated into a virus replicase complex. The exogenous genes under transcriptional control by the $26 \mathrm{~S}$ subpromoter combined with replicase complex would be largely transcribed in the cytoplasm and a large amount of double-stranded RNA would be produced $(8,27)$. Apoptosis of the host cell would then be induced in a short period to eliminate the risk of long-term existence of DNA fragments in cells and ensure safety (28). In vitro and in vivo experiments showed that when SL7207 was used as a vehicle, the pShT-GFP plasmid was effectively transferred into tumor cells and the GFP expression level was significantly higher in the SL/pShT-GFP group than in the SL/pIRES-GFP group. Meanwhile, the control WI-38 cells showed almost no GFP expression in vitro. Therefore, we believe that the plasmid pShT could targetly express exogenous genes in tumor cells and has higher levels of expression efficiency via SL7207 compared with ordinary vectors. 


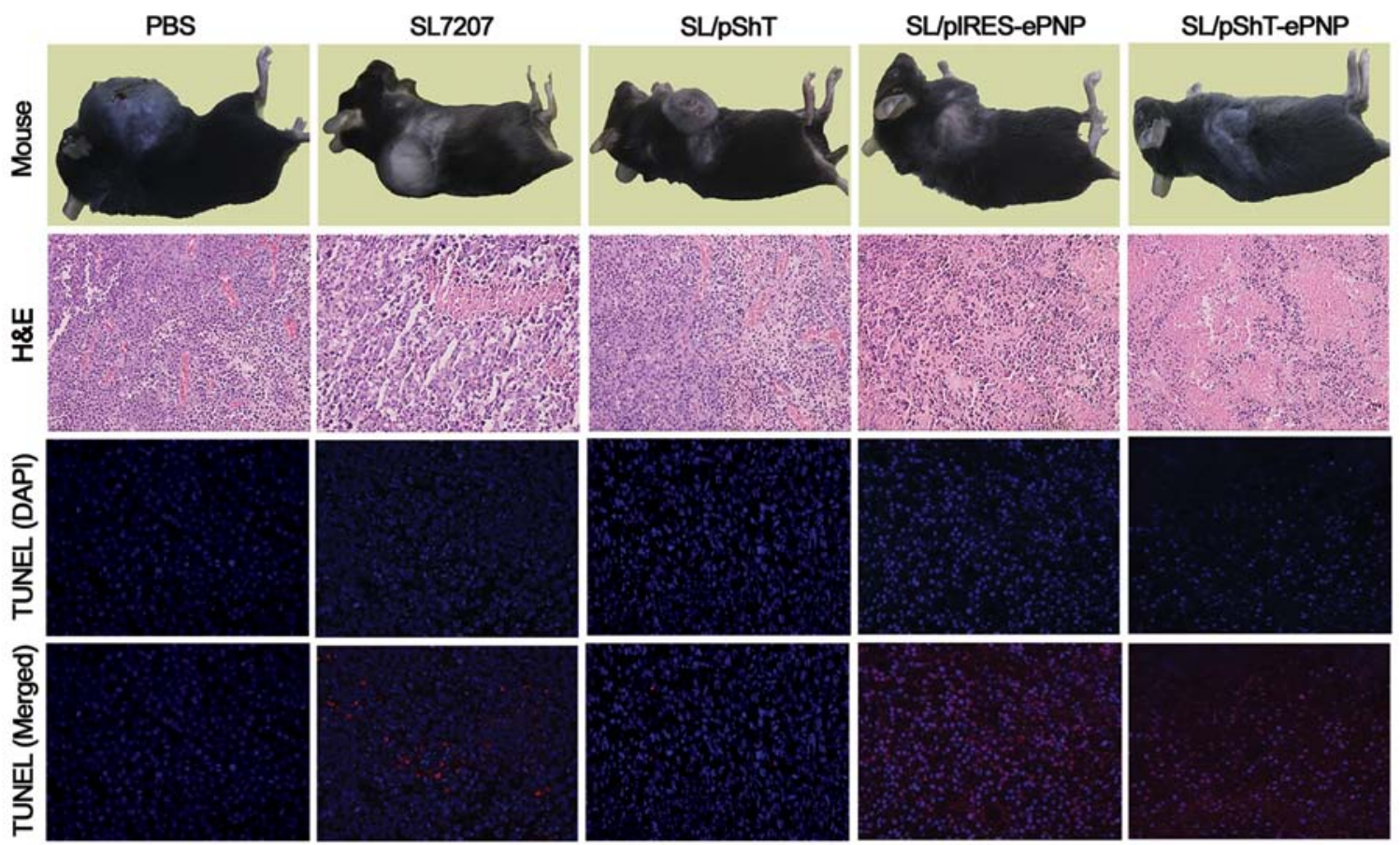

Figure 6. Histological analysis of B16 tumor xenografts from tumor-bearing mice treated with a different live recombinant bacterial vaccine plus F-dAdo at the end of the treatment cycle. Hematoxylin and eosin (H\&E) staining was performed. Original magnification, x200. Apoptotic tumor cells were detected using TUNEL staining. Original magnification, $\mathrm{x} 400$.
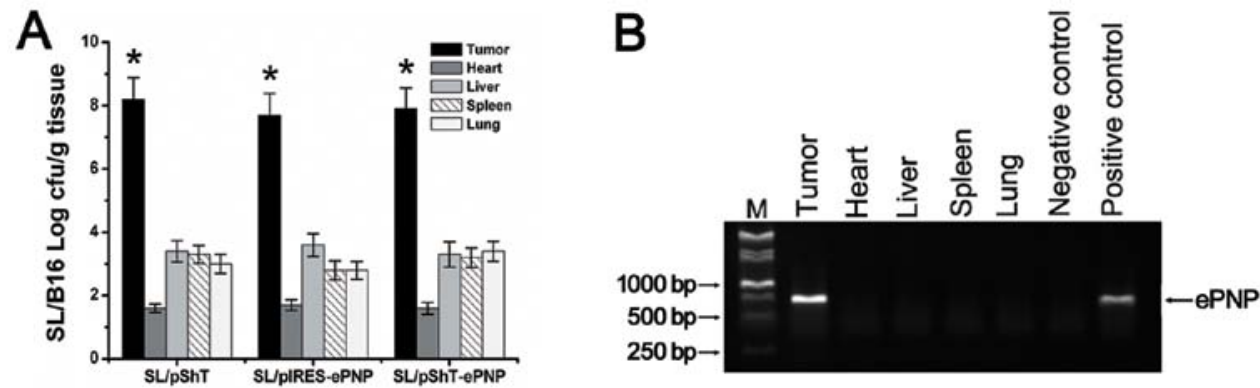

C
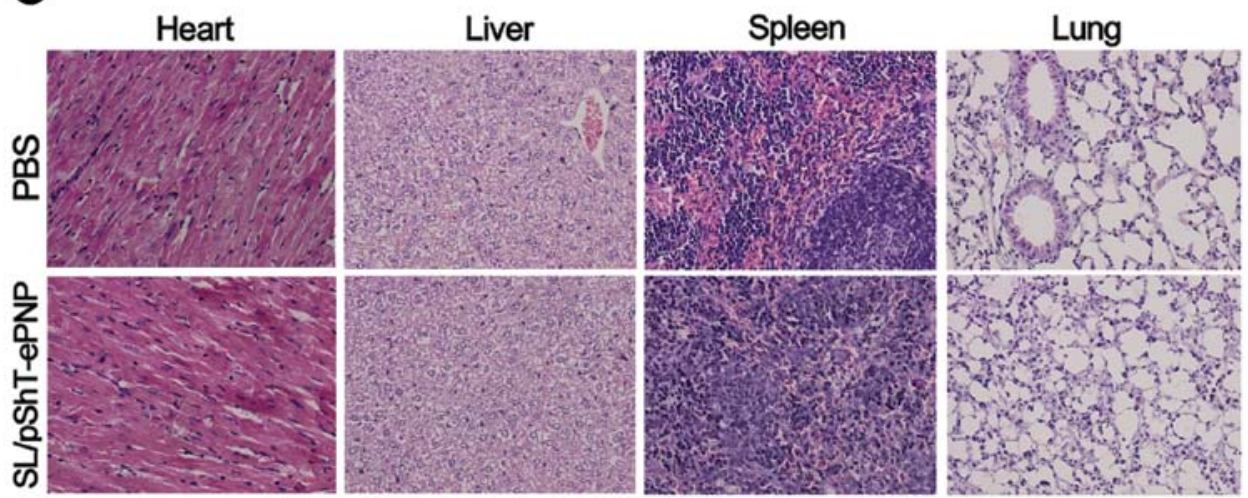

Figure 7. Targeted specificity and safety evaluation of the live attenuated Salmonella vaccine SL/pShT-ePNP. (A) On the fourth day of the first course, the number of SL7207 in tumor and important organs of B16 tumor-bearing mice in each group $(n=3)$ was determined. The data shown represent the mean \pm SD values. "P $<0.05$ versus the amount of bacteria in the liver. (B) On the fourth day of the first course, the expression of the ePNP gene in tumor tissue and various organs of B16 tumor-bearing mice in SL/pShT group ( $\mathrm{n}=3$ ) was detected by RT-PCR assays. (C) Tissues from the heart, liver, spleen and lung were extracted and used for histological analysis at the end of the treatment cycle. Original magnification, $\mathrm{x} 400$.

RT-PCR results also confirmed that the ePNP gene was expressed successfully in tumor cells in vitro. We found that when the MOI was 10 , the B16 cells viability was only $13.45 \%$.
However, when we used SL/pShT-GFP to infect B16 cells under the same conditions, flow cytometry showed that only $13.86 \%$ of the cells had GFP expression (data not shown). Combining 
these 2 results, we speculate that because the ePNP/F-Ado system has a powerful bystander effect, successful transfection of only a small fraction of cells would be sufficient to induce massive cell death $(1,29)$. We also observed that as the concentration of F-Ado and the treatment time increased, the cytotoxicity of the ePNP/F-Ado system proportionally increased. Additionally, HPLC results showed that F-Ado was effectively converted and could induce significant cytotoxicity from the early stage of treatment, possibly because the plasmid pShT expresses ePNP in a burst to effectively convert F-Ado at the early stage. These results show that the plasmid pShT-ePNP expresses ePNP specifically in tumor cells and in comparison with conventional CMV-promoter-based eukaryotic expression vectors, the expression efficiency is highly increased.

Because F-dAdo has a relatively short half-life (26), we chose to administer F-dAdo 2 days after oral administration of recombinant bacteria. F-dAdo was given 3 times a day for 3 days (18). At 4 days after oral administration of recombinant bacteria, the bacterial count in tumor tissues was $\sim 27,000$ times higher than that in the liver and other normal tissues, which indicates that SL7207 tends to aggregate in tumor tissues but still exists in normal tissues at a low concentration. The bacteria effectively aggregated in tumor tissues; however, because we applied the drug 2 days after oral administration of recombinant bacteria, we cannot exclude the possibility that other tissues were exposed to the cytotoxic effect. Therefore, to ensure clinical safety, we used the tumor-specific hTERTpromoter-containing pShT-ePNP plasmid (30). At the end of treatment, the safety evaluation indicated that SL/pShT-ePNP is highly safe because of its dual-level target specificity. The system effectively exerts a therapeutic effect on tumors; in particular, it may be effective for intratumoral sites that are not reachable by conventional chemoradiotherapy.

We found that SL/pShT-ePNP was effective in inhibiting tumor growth and prolonging the life of tumor-bearing mice; $\mathrm{H} \& \mathrm{E}$ staining and apoptosis assays also showed that tumors in the SL/pShT-ePNP group had significantly more necrosis and apoptosis than that in the other groups. We therefore speculate that the alphavirus replication enzyme compensated for the low efficiency of expression by hTERT in the pShT-ePNP therapeutic system. However, SL/pShT-ePNP did not cure the tumor despite its good therapeutic effect. We speculate that clearance of tumors relies on strong cytotoxic $\mathrm{T}$ lymphocyte responses that target tumor cells $(31,32)$. Therefore, in subsequent studies, we intend to use highly immunogenic proteins (such as Mycobacterium tuberculosis heat shock protein 70) or interleukin-12 to enhance the clearance of tumor cells $(33,34)$.

In conclusion, the recombinant live vaccines that we designed combined the advantages of various therapeutic vaccines reported in previous studies. We constructed a tumortargeted alphavirus replicon-based vector, pShT-ePNP, which highly expresses exogenous genes and delivered this plasmid into tumors by using attenuated $S$. typhimurium. In vitro and in vivo experiments both confirmed the high efficiency and high specificity of ePNP expression in tumor cells. When F-Ado was added, the ePNP/F-Ado system exerted significant therapeutic effects with respect to tumors. Our studies further confirmed a lack of adverse effects in SL/pShT-ePNP treated mice. Thus, we established that this vaccine is potentially useful for cancer therapy.

\section{Acknowledgements}

We gratefully thank every one of Pathology Department and Central Laboratory, Xiamen Maternal and Child Health Hospital for their sincere help. This study was supported by The National Natural Science Foundation of China (no. 30471603/ H1014).

\section{References}

1. Afshar S, Olafsen T, Wu AM and Morrison SL: Characterization of an engineered human purine nucleoside phosphorylase fused to an anti-her2/neu single chain Fv for use in ADEPT. J Exp Clin Cancer Res 28: 147, 2009.

2. Fukazawa T, Matsuoka J, Yamatsuji T, Maeda Y, Durbin ML and Naomoto Y: Adenovirus-mediated cancer gene therapy and virotherapy (Review). Int J Mol Med 25: 3-10, 2010.

3. Gu J, Kagawa S, Takakura M, Kyo S, Inoue M, Roth JA and Fang B: Tumor-specific transgene expression from the human telomerase reverse transcriptase promoter enables targeting of the therapeutic effects of the Bax gene to cancers. Cancer Res 60: 5359-5364, 2000.

4. Fakhoury J, Nimmo GA and Autexier C: Harnessing telomerase in cancer therapeutics. Anticancer Agents Med Chem 7: 475-483, 2007.

5. Song JS: Activity of the human telomerase catalytic subunit (hTERT) gene promoter could be increased by the SV40 enhancer. Biosci Biotechnol Biochem 68: 1634-1639, 2004.

6. Liljeström P and Garoff H: A new generation of animal cell expression vectors based on the Semliki Forest virus replicon. Biotechnology 9: 1356-1361, 1991.

7. Diciommo DP and Bremner R: Rapid, high level protein production using DNA-based Semliki Forest virus vectors. J Biol Chem 273: 18060-18066, 1998.

8. Kohno A, Emi N, Kasai M, Tanimoto M and Saito H: Semliki Forest virus-based DNA expression vector: transient protein production followed by cell death. Gene Ther 5: 415-418, 1998.

9. Quetglas JI, Ruiz-Guillen M, Aranda A, Cassales E, Bezunartea J and Smerdou C: Alphavirus vectors for cancer therapy. Virus Res 153: 179-196, 2010.

10. Wang WL, Xu HL, Lu RJ and Ruan L: A comparative study on SFV-based DNA vaccine and the conventional DNA vaccine. Chin J Virol 18: 325-331, 2002.

11. Juárez-Rodríguez MD, Arteaga-Cortés LT, Kader R, Curtiss R III and Clark-Curtiss JE: Live attenuated Salmonella vaccines against Mycobacterium tuberculosis with antigen delivery via the type III secretion system. Infect Immun 80: 798-814, 2012.

12. Hegazy WA and Hensel M: Salmonella enterica as a vaccine carrier. Future Microbiol 7: 111-127, 2012.

13. Loessner $\mathrm{H}$ and Weiss S: Bacteria-mediated DNA transfer in gene therapy and vaccination. Expert Opin Biol Ther 4: 157-168, 2004.

14. Jarosz M, Jazowiecka-Rakus J, Cichoń T, et al: Therapeutic antitumor potential of endoglin-based DNA vaccine combined with immunomodulatory agents. Gene Ther 12: 1038-1046, 2012.

15. Moreno M, Kramer MG, Yim L and Chabalgoity JA: Salmonella as live Trojan horse for vaccine development and cancer gene therapy. Curr Gene Ther 10: 56-76, 2010.

16. Sorscher EJ, Peng S, Bebok Z, Allan PW, Bennett LL Jr and Parker WB: Tumor cell bystander killing in colonic carcinoma utilizing the E. coli Deo D gene and generation of toxic purines. Gene Ther 1: 233-238, 1994.

17. Mosmann T: Rapid colorimetric assay for cellular growth and survival: application to proliferation and cytotoxicity assays. J Immunol Methods 65: 55, 1983.

18. Parker WB, Allan PW, Hassan AE, Secrist JA III, Sorscher EJ and Waud WR: Antitumor activity of 2-fluoro-2'-deoxyadenosine against tumors that express Escherichia coli purine nucleoside phosphorylase. Cancer Gene Ther 10: 23-29, 2003.

19. Wang W, Jin B, Li W, et al: Targeted antitumor effect induced by hTERT promoter mediated ODC antisense adenovirus. Mol Biol Rep 37: 3239-3247, 2010.

20. Hashimoto Y, Tazawa H, Teraishi F, et al: The hTERT promoter enhances the antitumor activity of an oncolytic adenovirus under a hypoxic microenvironment. PLoS One 7: e39292, 2012. 
21. Zhang J, Wild J, Bieler K and Graf M: Comparative study of expression and humoral immunogenicity of HIV-1 group specific antigen Pr55 (gag) by conventional plasmid vectors versus semliki forest virus-derived vectors. Chin J Virol 18: 529-536, 2002.

22. Brown A, Hormaeche CE, Demarco de Hormaeche R, Winther M, Dougan G, Maskell DJ and Stocker BA: An attenuated aroA Salmonella typhimurium vaccine elicits humoral and cellular immunity to cloned beta-galactosidase in mice. J Infect Dis 155: 86-92, 1987.

23. Hoiseth SK and Stocker BA: Aromatic-dependent Salmonella typhimurium are non-virulent and effective as live vaccines. Nature 291: 238-239, 1981.

24. Darji A, Guzman CA, Gerstel B, et al: Oral somatic transgene vaccination using attenuated S. typhimurium. Cell 91: 765-775, 1997.

25. Zeng S, Zhang J, Zhang J, et al: Suppression of murine melanoma growth by a vaccine of attenuated Salmonella carrying heat shock protein 70 and Herpes simplex virus-thymidine kinase genes. Oncol Rep 27: 798-806, 2012.

26. Fu W, Lan H, Li S, Han X, Gao T and Ren D: Synergistic antitumor efficacy of suicide/ePNP gene and 6-methylpurine 2'-deoxyriboside via Salmonella against murine tumors. Cancer Gene Ther 15: 474-484, 2008.

27. Leitner WW, Hwang LN, deVeer MJ, et al: Alphavirus-based DNA vaccine breaks immunological tolerance by activating innate antiviral pathways. Nat Med 9: 33-39, 2003.
28. Gil J, Alcami J and Esteban M: Induction of apoptosis by doublestranded-RNA-dependent protein kinase (PKR) involves the alpha subunit of eukaryotic translation initiation factor 2 and NF-kappaB. Mol Cell Biol 19: 4653-4663, 1999.

29. Xie X, Guo J, Kong Y, et al: Targeted expression of Escherichia coli purine nucleoside phosphorylase and Fludara ${ }^{\circledR}$ for prostate cancer therapy. J Gene Med 13: 680-691, 2011.

30. Ueki $\mathrm{H}$, Watanabe $\mathrm{M}$, Kaku $\mathrm{H}$, et al: A novel gene expression system for detecting viable bladder cancer cells. Int J Oncol 41: 135-140, 2012.

31. Ambade AV, Joshi GV and Mulherkar R: Effect of suicide gene therapy in combination with immunotherapy on antitumour immune response and tumour regression in a xenograft mouse model for head and neck squamous cell carcinoma. Indian J Med Res 132: 415-422, 2010.

32. Kuriyama S, Tsujinoue $\mathrm{H}$ and Yoshiji $\mathrm{H}$ : Immune response to suicide gene therapy. Methods Mol Med 90: 353-369, 2004.

33. Dong B, Sun L, Wu X, et al: Vaccination with TCL plus MHSP65 induces anti-lung cancer immunity in mice. Cancer Immunol Immunother 59: 899-908, 2010.

34. Yamazaki M, Straus FH, Messina M, et al: Adenovirus-mediated tumor-specific combined gene therapy using Herpes simplex virus thymidine/ganciclovir system and murine interleukin-12 induces effective antitumor activity against medullary thyroid carcinoma. Cancer Gene Ther 11: 8-15, 2004. 